\title{
INTEGRATED 3S TECHNOLOGY USED IN URBAN GRID MANAGEMENT
}

\author{
Hongrui Zhao ${ }^{\text {a, } * \text {, Hao Wang }}{ }^{\text {a }}$, Wenjia Wu ${ }^{\text {a }}$, Chaojun Wang ${ }^{\mathrm{a}}$ \\ a Institute of Geomatics, Department of Civil Engineering, Tsinghua University, Beijing, China; 3S Center, Tsinghua University, \\ Beijing, China-zhr@tsinghua.edu.cn, haowang90@gmail.com, wenjiajia411@msn.cn,1298373005@qq.com
}

Technical Commission II

KEY WORDS: Remote Sensing, Urban, Management, Extraction, Change Detection, GIS, GPS/INS

\begin{abstract}
:
Sustainable development requires monitoring the state and changes of the city and providing the appropriate information to users anytime and anywhere. This paper takes Ningbo City as the research area, by utilizing two temporal (March 25, 2012 and November $25,2013) \mathrm{ZY}-3$ satellite remote sensing data. 3S technology is used for urban grid management. The remote sensing information extraction of Ningbo City includes: extraction of building change information, extraction of green space change information, extraction of water information and analysis of eutrophication correspondingly. When extracting change information, we take "change information" as a special kind of "geographic information" to study the characteristics of different bands in multi-temporal data, and follow the first law of geography, namely adjacent similar principle. The extracted raster information is further converted into GIS vector format data as a basis for dynamic monitoring of Ningbo Urban Management systems: on the one hand, it can meet the demands of multi-source spatial data analysis (such as: overlay analysis, buffer analysis, etc.); on the other hand, it could meet the requirements of daily urban management. Dynamic monitoring system of Ningbo city management adopts the urban grid management mode. Based on GIS and GPS, grid management can satisfy the urban management mode -someone bear responsibility within the grid, somebody do the task under the supervision of the lattice, and at the same time play the role of remote sensing field surveying. To some extent, integrated $3 \mathrm{~S}$ technology and urban grid management is a practical alternative of minimizing the uncertainty of remote sensing data and information extraction. With multi-scale and multi-dimensional remote sensing data, $3 \mathrm{~S}$ integration and the urban grid management can monitor the urban state and its spatial-temporal changes. It's helpful for discovery and analysis of urban problems about resources, environment, ecology and disaster, from phenomena to nature, and it is a necessary part of sustainable urbanization.
\end{abstract}

\section{INTRODUCTION}

Sustainable development is an important issue in today's society, where sustainable urbanization is an important part and an important trend of future development. Joseph E. Stiglitz, American economist, Nobel laureate, said: "China's urbanization and new technological revolution in the United States were regarded as the two major events influencing the social process in 21st century" (Fan He, 2013). Promoting the new urbanization is a very complex system, and we have lots of contradictions and problems to tackle when facing urban development, especially in resource and environmental issues. For example: relative shortage of land resources, decreasing arable land; increasing urban water consumption, water pollution; serious environmental pollution problem, increasing ecological pressure. Hence, there is an urgent need for monitoring and managing the city's ecological environment.

Remote Sensing (Remote Sensing, RS) technology has the characteristics of large scale, real-time and repeatable data obtaining capacity, the information like buildings, water and vegetation can be extracted from remote sensing data; geographic information system (Geographic Information System, GIS) can process, dynamic access, manage and integrated service various sources of spatial data and attribute; global navigation and positioning system (Global Navigation Satellite System, GNSS) provide field research assistance and various types of location-based services through navigation; $3 \mathrm{~S}$ integration provides data sources and management platform for monitoring and managing sustainable cities.

Grid management is to divide the management zone into grid cells according to some kinds of standards, the grid cells act as the basic unit of the city administration. Through the grid scale, urban management can "fulfil their duties, complement each other, management according to the law, standard operation and quick response", thus improving city's level and efficiency of management.

Recently, 3S integration and grid management has provided new techniques and methods for urban management. But the most important thing is how to monitor the city through remote sensing, namely, how to accurately extract the required information from remote sensing data, including static information and changed information. As the existence of "different objects with same spectrum" and "same object with different spectra" existed in remote sensing, etc., together with the problem of insufficient information, there is great difficulty in information extraction.

\footnotetext{
* Corresponding author should be addressed to Hongrui Zhao, Email: zhr@tsinghua.edu.cn.
} 


\section{CHARACTERISTICS OF THE INFORMATION IN URBAN GRID MANAGEMENT}

\subsection{Contradiction between Uncertainty of Remote Sensing Data and Certainty of Information Needs of Urban Management}

Remote sensing data plays as the main data source. There is some uncertainty in remote sensing data for some reasons like mixed pixel and atmospheric effects, but also in remote sensing information extraction. However, urban grid management has a requirement for deterministic results, in another words, we have to ensure that both results from the space and the ground have to be exactly the same. Essentially, there is contradiction in scale, to be specific, the contradiction between the space scale and the ground scale.

\subsection{Contradiction between the Raster Data Format of Remote Sensing Data and the Vector Data Format of GIS Data}

The data format of remote sensing data is raster, which means that whatever information we get from remote sensing data should be raster data, whereas the foundation data in urban grid management is the vector GIS data, and the raster data is usually regarded as the background data. Though some software such as ENVI and ARCGIS enables people to convert vector and raster formats, the conversion is related to many problems such as cartographic generalization, object-oriented granularity, and attribute settings.

\subsection{Contradiction between Static Urban Morphology and} Dynamic Analysis of Ecological Environment

The analysis of city ecological environment requires both static data and dynamic data. Commonly, the static data is more available relative to the dynamic data, which refers to where, when and what change has occurred. Thus we have to construct the models to extract change information respectively.

\subsection{Contradiction between Spatial Analysis Data Requirements and Actual Data}

In order to fulfil the spatial analysis which ensures us to conduct process analysis and cause analysis of the ecological environment change, we have to consider the factors like standardized data format, unified mathematical foundation and consistent scale.

\section{INTRODUCTION OF NINGBO AND THE URBAN GRID MANAGEMENT SYSTEM}

Ningbo, also called "Yong" for short, is the second largest city in Zhejiang Province and known as many honors, such as the national historical and cultural city approved by Ministry of Culture of the People's Republic of China, one of the five regional centers in Yangtze River Delta, economic center of the south wing in Yangtze River Delta, the economic center of Zhejiang Province, an important international gateway to the Asia-Pacific region, a modern international port city, and three consecutive re-election civilized cities in China.

Ningbo (As shown in Figure 1) is located in the middle of the coastal line of the Chinese Mainland and in the South of the Yangtze River Delta. The longitude lies $120^{\circ} 55^{\prime}-122^{\circ} 16^{\prime} \mathrm{E}$ and latitude $28^{\circ} 51^{\prime}-30^{\circ} 33^{\prime} \mathrm{N}$. Ningbo is bounded on the east by the Zhoushan Archipelage, on the north by Hangzhou Bay, on the west by Shengzhou City, Xinchang City and Shangyu City of Shaoxing, on the south by Sanmen Bay, and connected with Sanmen City and Tiantai City of Taizhou. Moreover, Ningbo is also known as the southern port of the grand canal of China and the port of departure of the Maritime Silk Route.

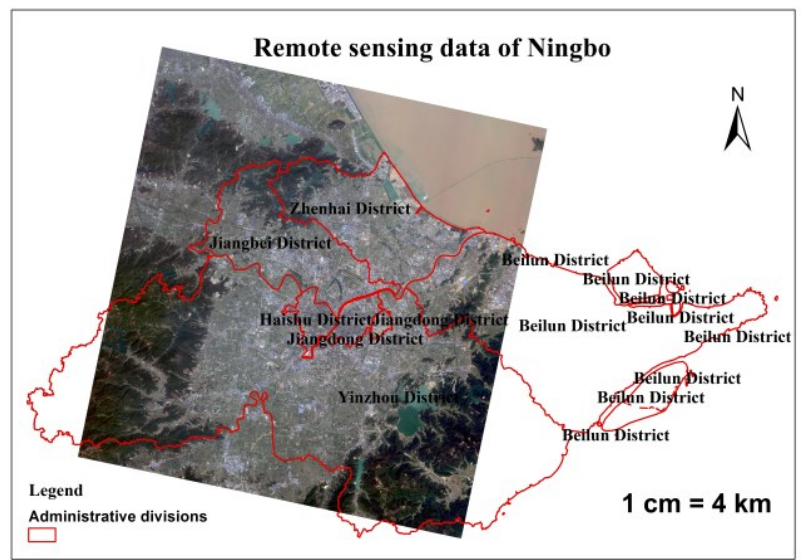

Figure 1. Remote sensing data of Ningbo (real colour composite image)

As for the ecological environment, Ningbo is one of the 8 drainage systems in Zhejiang Province, sandwiched between the ocean and low-lying mountains to the southwest. The ecological environment has great natural basis, socio-economic infrastructure and ecological foundation. However, the industrial and domestic pollution is serious causing blue-green algae, sediment and other water quality problems. The booming of construction projects and the decreasing of green space presents considerable challenges to the protection and construction of the ecological environment.

Thus there is a need to extract ecological information from remote sensing. Using the remote sensing data as main data resource, the urban grid management involves illegal construction supervision system, landscaping monitoring system, eutrophication monitoring system, sediment monitoring system and so on.

\section{MAIN TECHNIQUES}

\subsection{Geospatial Information Technology}

Geospatial information science and technology takes the 3S (RS, GIS and GNSS) as the main content. It is the discipline of gathering, measurement, analysis, storing, management, display, delivering, and application of geographic information, or spatially referenced information. It's a frontier of the Earth Science and is an important part of geo-information science. It is also the foundation of digital earth.

The core of sustainable urban management is the answer to $4 \mathrm{~W}$ (when, where, what object and what change) questions, and conducting 4A services (providing these spatial-temporal information anytime, anywhere to everyone, and service everything) (Deren Li, 2005). Therefore, the application of geospatial information technology for sustainable urban management is scientific and effective. However, new demands for urban management services raise new questions for geospatial information technology, such as remote sensing information extraction model, map generalization, and service mode. 
4.2 Remote Sensing Information Extraction Model and
Remote Sensing Information Extraction Results of Ningbo

Remote Sensing Data: two scenes of ZY3 multispectral data in 2012 and 2013.

Information Requirements: According to the characteristics and needs of Ningbo, when extracting information from remote sensing in Ningbo city, green spaces, water, and buildings should be extracted respectively, then analyse the changes of green spaces, water, buildings, as well as issues such as eutrophication of water bodies.

Information Extraction Model: Information extraction of green spaces, water and buildings has different characteristics.

The aim of green spaces extraction is to detect the change information. The conventional method is to extract green space information from the two temporal data separately, and then obtain the change information by subtracting. Due to the uncertainty of remote sensing data, it reveals a high errors percentage of omission and commission of extracted green spaces information extraction from single temporal, and results in greater uncertainty after subtraction. Based on the first law of geography "Everything is related to everything else, but near things are more related than distant things." we propose a new method, taking change information as a class feature. Combining the two temporal multi- spectral ZY3 data, a total of eight bands serve as the data source. By applying principal component analysis to the 8-band data, analysis and expression of change information is accomplished directly. At the same time, traditional subtraction change information extraction method is used together. By adjusting the thresholds, make sure there is no omission information in the subtraction results. Combining the subtraction results, the PCA information and other related information, using fuzzy classifiers, and the green spaces information is generated. The data format is raster which should be converted to GIS vector format afterwards. In the green space changing information of Ningbo, there are almost no new green spaces, as 2013 showed a relative decrease compared to 2012. But there remain extremely fractured patches in the extracted decrease information of green space, so the results cannot be directly converted to GIS vector data. The Decrease of green space in Ningbo from 2012 to 2013 is shown in Figure 2.

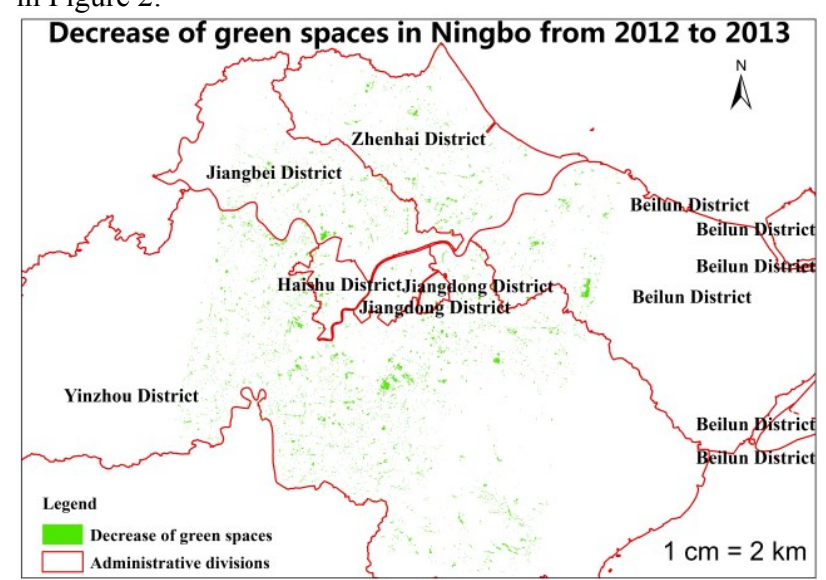

Figure 2. Decrease of green space in Ningbo from 2012 to 2013

The main aim of water extraction is observing the pollution of the water body, mainly manifesting as blue-green algae outbreaks and ultra-high sediments content. The spectral characteristics of the water body are quite different, easily confused with the spectral characteristics of the shadows and vegetation. With minimum noise fraction transformation (MNF Rotation) and other methods, water body information could be more prominent, but it is very difficult to get the information of small rivers, due to its weak and unclear nature. Combined with prior knowledge, using the hierarchical classification and partitioning method, taking good use of excessive classification and followed by post-processing method, assisted with decision tree and interactive methods, it can basically meet the requirements of the water body information extraction. Then calculate the content of blue-green algae and sediment on the basis of water extraction. The eutrophication and sediment monitoring results are shown in Figure 3.

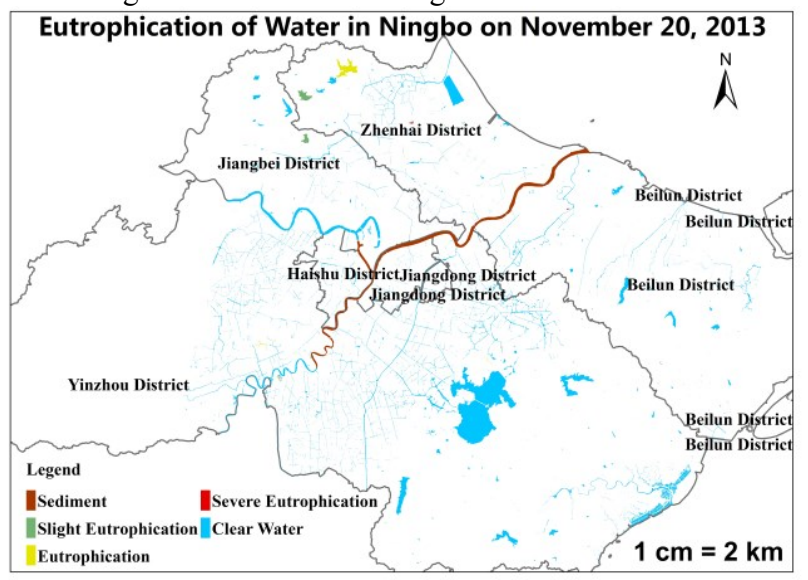

Figure 3. Eutrophication of Water Bodies in Ningbo on November 20, 2013

It is most difficult to extract information of the building from remote sensing image, as a variety of surface spectral features are similar to the spectra of the buildings, and the shadows have a great impact on building information extraction. The building patch itself is small and the results of automated building information extraction are far from ideal. So it is almost impossible to automatically extract the information of changed buildings and the construction sites. Human-computer interaction extraction is also very difficult, and it is also nearly impossible to observe and completely extract the change information based on the two temporal remote sensing data. By fusion of the two temporal data, the building change map can be generated. Human-computer interaction is helpful to extract the changed information based on the fused building change map. Figure 4 shows the changes of buildings in Ningbo from 2012 to 2013.



Figure 4. Changes of buildings in Ningbo from 2012 to 2013 


\subsection{Map Generalization}

Map generalization is required for the information extracted from remote sensing in order to meet the requirements of the GIS data used in city management. Map generalization removes and merges fine patches according to different spatial pattern conditions. Moreover, the remote sensing information extraction has the omission and excessive errors due to the uncertainty of remote sensing data. Excessive extraction can be removed through human-computer interaction during post-

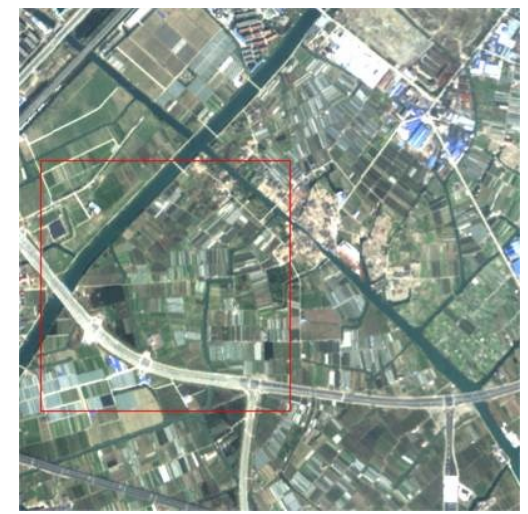

a. Multispectral ZY-3 data on March 25, 2012

(Mostly green spaces in the red box)

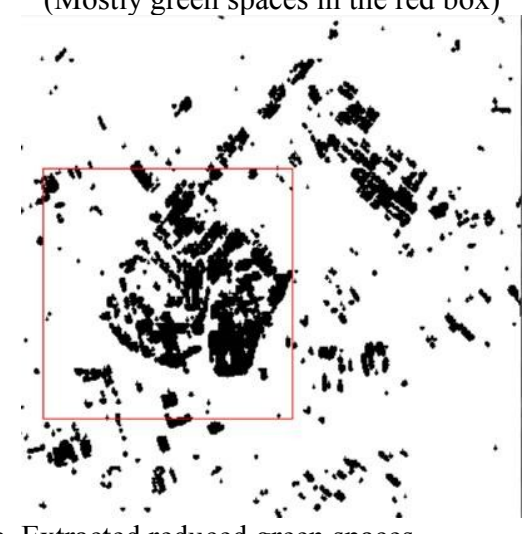

c. Extracted reduced green spaces

(Mostly fragmentized parcels in the red box) processing, but omission extraction seems unable to remedy. We propose the extraction principle of omission but not excessive. After that, expand neighbourhood buffer properly and merge the small patches spatially according to adjacent similar principle. Those still belong to fine patches will be removed after merging. The results will be transformed to GIS data, then check and correct the data topologically. Figure 5 shows the scheme of map generalization of the decrease of green spaces in Ningbo.

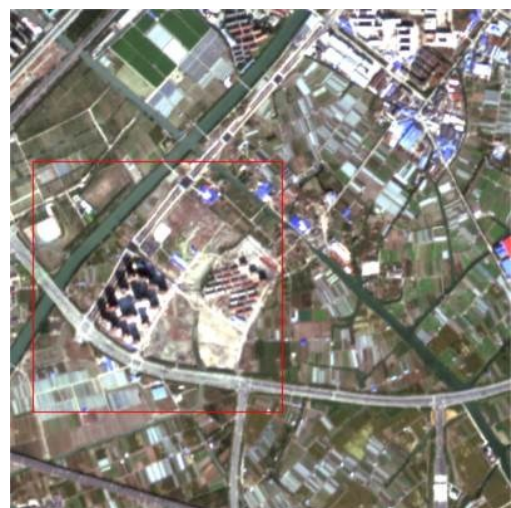

b. Multispectral ZY-3 data on November 20, 2013

(Green spaces decreased in the red box)

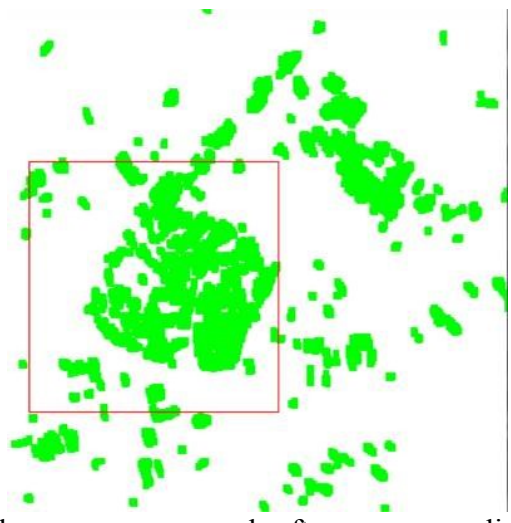

d. Decreased green spaces parcels after map generalization (fragmentized parcels decreased in the red box)

Figure 5. A scheme showing the map generalization of the decrease of green spaces in Ningbo

\subsection{Urban Management and Service Mode}

Urban grid management is presented and first pilot by Dongcheng district, Beijing city, China, in October 2004. It is characterized as digital, detailed, real-time and closed-loop management, and the efficiency of urban management improved significantly.

Grid management is a kind of multi-scale management. It forms 4 progressive, gradually refined management scales in space. Take Dongcheng District of Beijing as an example (Jiang and Ren, 2007), scale 1 refers to the entire Dongcheng District, District Government is responsible for city management; scale 2 refers to 10 streets, Street Offices take responsibility; scale 3 refers to 137 communities, Community committees take responsibility; scale 4 refers to grid units, resident units and local people take responsibility. Manage parts of the city such as manhole covers, street lamps in the grid scale, etc. Multiscale management makes the processing time accurate to seconds.

In grid management mode, information extracted from remote sensing need to be converted into some certain data formats to facilitate grid management, and restored in the grid management database. Generally, the built-in topographic maps of grid management terminal "Cheng Guan Tong " are large-scale topographic maps, whereas remote sensing mapping is limited by spatial resolution of remotely sensed data. In order to extract urban water bodies, vegetation and other multi-dimensional information, a certain spectral resolution is needed, while the spatial resolution and spectral resolution is contradictory. Thus, required information of urban management extracted from remote sensing cannot comply with the accuracy requirements of large scale surveying and mappings, but need to proceed 
from the needs of urban management, combine the remote observation scale with that of the ground grid management, and achieve the ground verification and map investigation through the grid management. Thus, on the one hand remote sensing serves as a valid data source of urban management, it can provide important information which alternative are difficult to obtain; on the other hand, urban grid management overcomes the uncertainty of remote sensing data in a large extent. Take the advantages of "Space (Remote Sensing)" and "Ground (urban grid management)", $3 \mathrm{~S}$ integrated urban grid management could do something on sustainable urban management.

\section{CONCLUSIONS}

3S integration and urban grid management can take full advantage of multi-scale multi-dimensional remote sensing data, monitor the status and spatial-temporal evolution of cities. It can help the analysis of cities from phenomenon to nature, finding the general problems faced by cities, such as resources, environment, ecology, disaster and other issues. It is an essential component of sustainable urbanization.

\section{ACKNOWLEDGEMENTS}

The study has been supported by the Special Research Project for the Commonwealth of the Ministry of Water Resources of the People's Republic of China (Grant No. 201201092).

\section{REFERENCES}

He, F.,2013. "China's coming urbanization is also a revolution in the administrative system of governance",Xinhuanet.http://news.xinhuanet.com/english/inde pth/2013-01/25/c_132616688.htm (25 Jan. 2014).

Li, D.R., 2005. Geo-spatial Information Science and It's Applications in Land Science. Chinese Journal of Nature, 27(6), pp. 316-322.

Jiang, A.L. and Ren, Z.R., 2007. Grid Management: New Mode of Modern Urban Management--research on certain questions of urban grid management mode. Shanghai Urban Planning Review, 72(1), pp.9-11. 